\title{
Dosage compensation in high resolution: global up-regulation through local recruitment
}

\author{
Dirk Schübeler ${ }^{1}$ \\ Friedrich Miescher Institute for Biomedical Research, 4058 Basel, Switzerland
}

Dosage compensation solves the chromosomal imbalance that is a result of sexual determination by sex chromosomes. It equalizes gene expression between the homogametic $(\mathrm{XX})$ and heterogametic $(\mathrm{XY})$ sexes and thus needs to selectively modify expression from the $\mathrm{X}$ chromosome in a sex-specific manner without affecting transcription on the autosomes. Various strategies have evolved in different organisms to achieve this balance, and their study has contributed significantly to our understanding of transcriptional gene regulation of whole chromosomes and established several paradigms of epigenetic control (Lucchesi 1998; Stuckenholz et al. 1999; Akhtar 2003).

In mammals, dosage compensation is accomplished by inactivating one copy of the $\mathrm{X}$ chromosome in females via an epigenetic process of allele-specific modification of chromatin and DNA. In Drosophila, dosage compensation is achieved not by repression but by increasing the transcription specifically on the single male X chromosome (Hamada et al. 2005; Straub et al. 2005). Genetic screens for male-specific lethality (MSL) identified five protein-coding genes that are required for dosage compensation: Msl 1-3, male absent on the first (mof), and maleless (mle). Subsequent biochemical characterizations suggested that these proteins, together with two noncoding RNAs (roX1 and roX2), form what has been termed the dosage compensation complex (DCC) (for review, see Bashaw and Baker 1996; Gilfillan et al. 2004). Complex formation only occurs in males, as translation of the MSL-2 protein is inhibited in females.

A first evidence for chromatin as a target in dosage compensation came from the observation of higher levels of histone H4K16 acetylation on the hyperactivated X detected by immunostaining (Turner et al. 1992). One of the msl genes, MOF, is a histone acetyl-transferase (HAT) that acetylates $\mathrm{H} 4$ at Lys 16 and is able to cause derepression of chromatinized templates in vitro and in

${ }^{1}$ Correspondence.

E-MAIL dirk@fmi.ch; FAX 41-61-6973976.

Article published online ahead of print. Article and publication date are at http://www.genesdev.org/cgi/doi/10.1101/gad.1423006. vivo (Akhtar and Becker 2000). Thus, it appears that the HAT activity of MOF plays an important part in the mechanisms that lead to hyperactivation of the male $\mathrm{X}$ (Smith et al. 2000).

A large body of work in different systems established that histone hyperacetylation correlates with gene activation, making this a feasible model (Wade et al. 1997). Yet how does the recruitment of a HAT activity that acetylates a single lysine on $\mathrm{H} 4$ result in a precise twofold up-regulation of mRNA? As histone acetylation is involved in promoter activation, it has been assumed that DCC is recruited to promoters of genes at the X chromosome, but is this really the site of action in vivo? Are individual genes targeted by DCC, or are large chromosomal regions covered? Equally as important, how does this process ensure regulation of X-linked genes that are dynamically expressed during development? Is compensation set up early in development for all genes independent of their subsequent activity, or is the DCC relocated dynamically to any activated gene? Many of these questions can be approached by defining sites and kinetics of DCC recruitment on the X chromosome in high spatial resolution at different developmental time points. No less than three reports in this issue of Genes \& Development provide such chromosome-wide analysis of several MSL proteins. Together they provide important and unexpected insights into the process of dosage compensation, challenging and helping to redefine current models (Alekseyenko et al. 2006; Gilfillan et al. 2006; Legube et al. 2006).

\section{Local recruitment-chromosome-wide up-regulation?}

Historically, DCC binding has been studied by immunostaining of polytene chromosomes in salivary glands. These giant polytene chromosomes are the result of several rounds of endoreplication leading to an estimated 500 copies of most euchromatic regions of the genome. The copies coalign and can be analyzed by light microscopy. The DCC can be detected on polytene chromosomes at hundreds of sites on the $\mathrm{X}$ chromosome in males (Demakova et al. 2003). This association and re- 
sulting hypertranscription of the male $\mathrm{X}$ chromosome require an intact DCC including proteins and noncoding RNAs (Gu et al. 1998). Deletion of either MOF, MLE, or MSL-3 results in the formation of a partial complex, which still localizes to the $\mathrm{X}$ chromosome, yet only at a small ( 35) number of positions (Kelley et al. 1999). This observation has led to the model that these high-affinity sites provide entry points for the intact complex, which precedes a spreading along the chromosome. Mutants in the catalytic domains of MOF or MLE also show no widespread binding, suggesting that hyperacetylation by MOF and the helicase activity of MLE are required to bind low-affinity sites (Gu et al. 2000). Interestingly, MOF binding to the $\mathrm{X}$ chromosome requires interaction with RNA (Akhtar et al. 2000)-presumably roX1 and roX2 (Meller and Rattner 2002). These noncoding RNAs are also required for correct targeting in support of a model in which the DCC is recruited by X-specific sequences. Indeed, regions of the $\mathrm{X}$ chromosome still recruit the DCC when translocated to autosomes (Kelley et al. 1999). Recent studies are starting to challenge the model of complex spreading subsequent to binding to high-affinity sites, since many X-derived translocations were able to ectopically recruit the complex to autosomes (Fagegaltier and Baker 2004). In addition, translocation of high-affinity sites to autosomal regions did not necessarily result in spreading (Oh et al. 2004). Alternative models for specific recruitment, therefore, propose large numbers of different affinity sites that together mediate local targeting (Dahlsveen et al. 2006).

The above-described localizations were identified on polytene chromosomes with the limited resolution of light microscopy. The three reports from the groups of Akhtar (Legube et al. 2006), Becker (Gilfillan et al. 2006), and Kuroda (Alekseyenko et al. 2006) now provide chromosome-wide localization information for different proteins of the DCC in an unprecedented resolution. Moreover, between these studies, binding of DCC members has been mapped in vivo at different developmental stages and in cell culture models, resulting in extensive data sets. All three studies use chromatin immunoprecipitation (ChIP) to enrich DCC protein-bound sequences with subsequent detection by DNA arrays (ChIP-chip). The high genomic coverage and spatial resolution of the chosen microarrays allow quantitative analysis of chromosomal characteristics and sequence features of DCC-binding sites.

Alekseyenko et al. (2006) use ectopic expression of epitope-tagged MSL-3 (MSL-3-TAP) and use the epitope to purify MSL-3 targets. MSL-3-TAP rescues msl3 mutant males and shows a similar polytene distribution, suggesting that tagging and ectopic expression do not interfere with DCC function and localization (Alekseyenko et al. 2006). Subsequently, ChIP-chip experiments are performed from stably transfected tissue culture cells or fly embryos hybridized to an oligo array. The chosen microarray covers the complete $\mathrm{X}$ chromosome and most of the left arm of chromosome 2 (2L), which serves as an autosomal region that should not be dosage-compensated. On the other hand, Gilfillan et al. (2006) per- form ChIP against endogenous MSL-1 in fly embryos and use either a comparable chromosomal tiling array or a microarray representing only genic regions as individual cDNA clones. In the third study, Legube et al. (2006) performed ChIP against endogenous MSL-1 and MSL-3 using fly embryos or isolated salivary glands as starting material, and the detection is performed on a microarray consisting of cDNA clones. The use of cDNA arrays allows the screening of more individual genes but by definition gives no direct information on the distribution at intergenic regions and is unlikely to reveal preferential localization along a particular gene, as a single probe represents each gene.

Despite these differences in experimental tools and systems, all three studies show remarkable similarity in the general observation that all tested DCC members bind almost exclusively to a selected group of genes on the $\mathrm{X}$ chromosome. Altogether, 739 binding regions (defined as clusters of neighboring oligomers that show enrichment) are identified for MSL-3 (Alekseyenko et al. 2006), and 773 for MSL-1 (Gilfillan et al. 2006). We can assume that these two groups of target genes are largely overlapping, as the third study shows a high similarity between MSL-1- and MSL-3-bound genes on a cDNA array (Legube et al. 2006). Thus a surprisingly large number of MSL-binding sites can be identified on the X chromosome in nonpolyploid cells, and they are likely to be bound by all tested proteins, suggesting that in each case the complete DCC complex is detected.

Binding is observed at discrete regions, and together $<25 \%$ of the $\mathrm{X}$ chromosome appears to be bound by the DCC (Gilfillan et al. 2006). This observed pattern indicates that DCC recruitment leads to local binding and does not coat chromosomal regions. Indeed, binding occurs with high preference at genes and not at intergenic sequences. This is apparent if selected chromosomal regions are inspected visually, and is further validated by statistical analysis showing that the vast majority (>90\%) of enriched sequences for MSL-3-TAP (Alekseyenko et al. 2006) and MSL-1 (Gilfillan et al. 2006) are genic. A comparison of binding with expression state shows that mostly active genes and not inactive genes are DCC targets. This holds true in fly embryos, in an embryo-derived cell line (SL2), and in a cell line derived from larvae imaginal discs (Clone8). Importantly, while DCC presence indicates expression state, it does not appear to reflect the level of transcription, implying a certain threshold for DCC binding (Alekseyenko et al. 2006; Gilfillan et al. 2006; Legube et al. 2006). Furthermore, not all active genes are targets, indicating that transcription alone is insufficient to recruit the complex (see below).

If identified DCC targets are indeed dosage-compensated, they should be identically expressed between males and females and loss of the complex should lead to their transcriptional down-regulation. This expected twofold change in expression is difficult to measure, but nevertheless knockdown of MSL-2 in SL2 cells reduces expression preferentially at DCC target genes (Alekseyenko et al. 2006; Gilfillan et al. 2006), suggesting that in 
this cell type, DCC binding is indicative of dosage compensation.

\section{Developmental dynamics of DCC binding}

The finding that MSL proteins are present at the majority of active genes poses the question: Does DCC recruitment follow expression changes, and thus is it dynamic during development? A comparison of the MSL-3-binding profiles between two tissue culture cells (SL2 and Clone8) and 12- to 17-h embryos shows high similarity in binding, suggesting strong conservation of binding sites (Alekseyenko et al. 2006). Notably, the transcriptional profiles are also remarkably similar. Yet a small subset of genes that is only expressed in either Clone8 or SL2 cells was tested individually and showed MSL-3 binding only in the cell where a gene was active (Alekseyenko et al. 2006). This indicates that DCC binding can be dynamic between cell types, and it will be interesting to see if these differences can also be observed between primary cells from early embryos (from which SL2 cells are derived) and larvae imaginal discs (from which Clone8 cells are derived).

Strikingly, a comparison of MSL-1 binding between 4to 6-h-staged embryos and larvae salivary gland cells reveals only very minor differences in DCC binding, while a large number of expression changes can be detected (Legube et al. 2006), suggesting early establishment of DCC binding. This is supported by a previous comparison of polytene staining at different larval stages, which showed stable binding of DCC independent of transcriptional changes (Kotlikova et al. 2006). Moreover, expression profiles of male and female salivary glands show only a modest correlation between dosage compensation, measured as equal expression in male and female, and DCC binding (Legube et al. 2006). Of the cell types analyzed, only the salivary gland cells are terminally differentiated, while embryonic and imaginal disc cells are multipotent and mitotically dividing, opening the possibility that DCC binding is not dynamic past a certain developmental state. Such regulated plasticity could be linked to cellular potency and be more restricted in terminally differentiated cells. It will be important to investigate if this reflects changes in the ability to recruit DCC versus maintaining its presence on the chromosome.

\section{Up-regulation by binding downstream of the promoter}

The observed discrete binding to active genes itself is compatible with models of transcriptional up-regulation by the DCC. Current concepts of transcriptional regulation focus on the promoter as the determining element for gene activity and rate of transcription. One of the most surprising observations in the newly published studies is the strong abundance of DCC proteins at the middle and $3^{\prime}$ end of target genes and the coincident weak binding of promoter sequences (Fig. 1A; Alekseyenko et al. 2006; Gilfillan et al. 2006). This bias is universally observed and is reflected in a $3^{\prime}$-biased distribu- tion of H4K16 acetylation at selected genes that have been analyzed individually (Smith et al. 2001; Gilfillan et al. 2006). Together, this suggests that the DCC is not primarily acting at the promoter, but downstream of polymerase initiation. This is further supported by the finding that the levels of bound polymerases are not increased on the hyperactivated X chromosome /Gilfillan et al. 2006), as might be expected if initiation is the main target. How can binding at the $3^{\prime}$ part of a gene result in a twofold up-regulation of mRNA levels? Passage of the polymerase through chromatin requires a large number of elongation factors, many of which remodel or modify chromatin (Sims et al. 2004). Thus, it is conceivable that a chromatin change, such as the DCC-mediated acetylation of $\mathrm{H} 4$, could have a positive effect on the elongation rate of polymerase. However, in a simple model of transcription, in which initiation, elongation, and termination are independent events on a linear template, an increase in polymerase processivity would have little effect on overall mRNA levels, as in most cases initiation will be the rate-limiting step (Fig. 1B). For example, in nuclear run-on experiments, in which polymerase density is measured by releasing transcription in the absence of chromatin, only a few genes show signs of paused polymerases and thus appear to be regulated by elongation (Rougvie and Lis 1990). Nevertheless, an increased elongation rate could have a direct effect on the initiation rate if both events are connected; for example, if polymerases can reinitiate and "recycle" at the same gene. This would require that $3^{\prime}$ and $5^{\prime}$ ends are in close physical proximity (Fig. 1B). Indeed, there is emerging evidence from budding yeast supporting a direct crosstalk and spatial proximity between the $3^{\prime}$ and $5^{\prime}$ untranslated regions (UTRs), required for high-level transcription (O'Sullivan et al. 2004; Ansari and Hampsey 2005).

Alternatively, DCC binding to the $3^{\prime}$ end could lead to more effective mRNA processing and/or increased stability. This could be mediated by recruitment of specific factors or by DCC-mediated nuclear relocalization of the
A

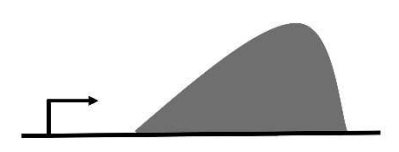

B
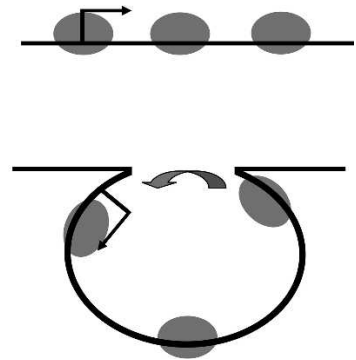

Figure 1. (A) Schematic distribution of MSL members across a typical gene that is dosage-compensated on the male X chromosome of Drosophila. The arrow indicates the transcriptional start site. MSL binding is low at the promoter and increases to the $3^{\prime}$ end. $(B)$ Linear and circular model of transcription. Polymerase is indicated in gray. If $3^{\prime}$ and $5^{\prime}$ UTRs interact physically, a terminating polymerase could reinitiate at the same gene. In such a scenario, any increase in elongation speed would raise mRNA levels. 
gene to a compartment that enhances transcriptional initiation or RNA processing. Any of these models is testable by investigating the $3 \mathrm{D}$ organization of dosage-compensated genes.

\section{Targeting of DCC}

Sequence determinants for DCC recruitment have been a mystery. The availability of high-resolution and comprehensive data sets of DCC-targeted genes should allow one to gain insights into sequence features that distinguish targets from nontargets. Two studies attempt this goal with bioinformatics approaches, and both identify certain sequence motifs to be more abundant at dosagecompensated genes. Akhtar and colleagues (Legube et al. 2006) analyze promoter motifs of target and nontarget genes and find a significantly higher incidence of a DNA replication element factor (DREF)-binding motif, suggesting that this sequence motif might aid in dosage compensation. However, DREF elements are only slightly more abundant at target genes, and the motif itself is very frequent at Drosophila promoters (Ohler et al. 2002), making it unlikely that DREF alone could direct DCC recruitment. Becker and colleagues (Gilfillan et al. 2006) used partial least squares regression (PLSR) on the complete chromosomal profile to identify sequence motifs with potential to predict MSL-1 binding. They identify an extensive set of hexamer motifs, which they show to have noticeable, yet limited, power in predicting MSL binding (Gilfillan et al. 2006). Further indepth analysis of the complete data sets from all studies should allow one to test and possibly expand these initial observations of underlying sequence components. The apparent complexity of identifying DCC recruitment sites based on sequence is reminiscent of identifying polycomb response elements (PRE), a group of cis-acting elements that modify transcriptional activity through chromatin (Ringrose and Paro 2004). In the case of PRE, predictions were only made possible after extensive in vivo definition of several active elements and their use as a training set in subsequent computational approaches (Ringrose et al. 2003).

The limited ability to predict DCC binding from a single sequence motif is unlikely to reflect only the limits of current computational approaches, but rather indicates that binding occurs through integration of multiple weak interactions between DCC members and sequences proximal to compensated genes. Such weak interactions are probably infrequent and short-lived but could be sufficient to increase local concentrations of the DCC complex or individual members. In combination with a second recognized signal, this could lead to stable binding. As the DCC targets the 3' end of active genes, this second interaction partner could be a transcriptiondependent protein component, such as an elongation factor or modified chromatin.

The genomic distributions of a large number of covalent histone modifications have been described recently (Schübeler et al. 2004; Liu et al. 2005; Pokholok et al. 2005). Among these, only methylation of Lys 36 of his- tone H3 appears to be preferentially enriched downstream of the promoter as suggested by genome-wide analysis in Saccharomyces cerevisiae (Pokholok et al. 2005; Rao et al. 2005) and single-gene analysis in chicken erythrocytes (Bannister et al. 2005). In S. cerevisiae, K36 methylation recruits histone-deacetylase (HDAC) activity via the chromodomain protein eaf-3 (Carrozza et al. 2005; Joshi and Struhl 2005; Keogh et al. 2005) and thus has been postulated to be involved in chromatin compaction following passage of the polymerase complex. Interestingly, MSL-3 represents one of the two Drosophila homologs of eaf-3, and thus it is tempting to speculate that it might interact via its chromo-domain with methylated Lys 36 of histone H3 (Alekseyenko et al. 2006). The chromosomal distribution of K36 and the responsible histone methyltransferase(s) have so far not been described for Drosophila, leaving open the question of how much of this chromatin pathway is conserved in flies.

Importantly, H4K16 hyperacetylation has been detected at sites of DCC binding at promoter-distal regions (Smith et al. 2001; Gilfillan et al. 2006). Thus, it is conceivable that DCC recruitment could antagonize K36mediated deacetylation of reading frames by mediating acetylation of H4K16. While this potential cross-talk between K36 methylation and DCC remains to be shown, it could represent an unexpected additional role for chromatin in the process of dosage compensation. Not only would a histone residue be modified by DCC, but a different modified residue could be involved in complex targeting in conjunction with X-chromosome-specific sequences.

\section{Acknowledgments}

I thank Antoine Peters, Michaela Schwaiger, Asifa Akhtar, Peter Becker, Gregor Gilfillan, and Mitzi Kuroda for comments on the manuscript; in particular, Oliver Bell for important insights into the subjects discussed. Work in my laboratory is supported by the Novartis Research Foundation.

\section{References}

Akhtar, A. 2003. Dosage compensation: An intertwined world of RNA and chromatin remodelling. Curr. Opin. Genet. Dev. 13: $161-169$.

Akhtar, A. and Becker, P.B. 2000. Activation of transcription through histone $\mathrm{H} 4$ acetylation by MOF, an acetyltransferase essential for dosage compensation in Drosophila. Mol. Cell 5: 367-375.

Akhtar, A., Zink, D., and Becker, P.B. 2000. Chromodomains are protein-RNA interaction modules. Nature 407: 405-409.

Alekseyenko, A.A., Larschan, E., Lai, W.R., Park, P.J., and Kuroda, M.I. 2006. High-resolution ChIP-chip analysis reveals that the Drosophila MSL complex selectively identifies active genes on the male X chromosome. Genes \& Dev. (this issue).

Ansari, A. and Hampsey, M. 2005. A role for the CPF 3 '-end processing machinery in RNAP II-dependent gene looping. Genes \& Dev. 19: 2969-2978.

Bannister, A.J., Schneider, R., Myers, F.A., Thorne, A.W., Crane- 
Robinson, C., and Kouzarides, T. 2005. Spatial distribution of di- and tri-methyl lysine 36 of histone $\mathrm{H} 3$ at active genes. J. Biol. Chem. 280: 17732-17736.

Bashaw, G.J. and Baker, B.S. 1996. Dosage compensation and chromatin structure in Drosophila. Curr. Opin. Genet. Dev. 6: 496-501.

Carrozza, M.J., Li, B., Florens, L., Suganuma, T., Swanson, S.K., Lee, K.K., Shia, W.J., Anderson, S., Yates, J., Washburn, M.P., et al. 2005. Histone H3 methylation by Set 2 directs deacetylation of coding regions by Rpd3S to suppress spurious intragenic transcription. Cell 123: 581-592.

Dahlsveen, I.K., Gilfillan, G.D., Shelest, V.I., Lamm, R., and Becker, P.B. 2006. Targeting determinants of dosage compensation in Drosophila. PLoS Genet. 2: e5.

Demakova, O.V., Kotlikova, I.V., Gordadze, P.R., Alekseyenko, A.A., Kuroda, M.I., and Zhimulev, I.F. 2003. The MSL complex levels are critical for its correct targeting to the chromosomes in Drosophila melanogaster. Chromosoma 112: 103-115.

Fagegaltier, D. and Baker, B.S. 2004. X chromosome sites autonomously recruit the dosage compensation complex in Drosophila males. PLoS Biol. 2: e341.

Gilfillan, G.D., Dahlsveen, I.K., and Becker, P.B. 2004. Lifting a chromosome: Dosage compensation in Drosophila melanogaster. FEBS Lett. 567: 8-14.

Gilfillan, G.D., Straub, T., de Wit, E., Greil, F., Lamm, R., van Steensel, B., and Becker, P.B. 2006. Chromosome-wide genespecific targeting of the Drosophila dosage compensation complex. Genes \& Dev. (this issue).

Gu, W., Szauter, P., and Lucchesi, J.C. 1998. Targeting of MOF, a putative histone acetyl transferase, to the $\mathrm{X}$ chromosome of Drosophila melanogaster. Dev. Genet. 22: 56-64.

Gu, W., Wei, X., Pannuti, A., and Lucchesi, J.C. 2000. Targeting the chromatin-remodeling MSL complex of Drosophila to its sites of action on the $\mathrm{X}$ chromosome requires both acetyl transferase and ATPase activities. EMBO J. 19: 5202-5211.

Hamada, F.N., Park, P.J., Gordadze, P.R., and Kuroda, M.I. 2005. Global regulation of X chromosomal genes by the MSL complex in Drosophila melanogaster. Genes \& Dev. 19: 22892294.

Joshi, A.A. and Struhl, K. 2005. Eaf3 chromodomain interaction with methylated H3-K36 links histone deacetylation to Pol II elongation. Mol. Cell 20: 971-978.

Kelley, R.L., Meller, V.H., Gordadze, P.R., Roman, G., Davis, R.L., and Kuroda, M.I. 1999. Epigenetic spreading of the Drosophila dosage compensation complex from roX RNA genes into flanking chromatin. Cell 98: 513-522.

Keogh, M.C., Kurdistani, S.K., Morris, S.A., Ahn, S.H., Podolny, V., Collins, S.R., Schuldiner, M., Chin, K., Punna, T., Thompson, N.J., et al. 2005. Cotranscriptional set2 methylation of histone $\mathrm{H} 3$ lysine 36 recruits a repressive Rpd3 complex. Cell 123: 593-605.

Kotlikova, I.V., Demakova, O.V., Semeshin, V.F., Shloma, V.V., Boldyreva, L.V., Kuroda, M.I., and Zhimulev, I.F. 2006. The Drosophila dosage compensation complex binds to polytene chromosomes independently of developmental changes in transcription. Genetics 172: 963-974.

Legube, G., McWeeney, S.K., Lercher, M.J., and Akhtar, A. 2006. X-chromosome-wide profiling of MSL-1 distribution and dosage compensation in Drosophila. Genes \& Dev. (this issue).

Liu, C.L., Kaplan, T., Kim, M., Buratowski, S., Schreiber, S.L., Friedman, N., and Rando, O.J. 2005. Single-nucleosome mapping of histone modifications in S. cerevisiae. PLoS Biol. 3: e328.

Lucchesi, J.C. 1998. Dosage compensation in flies and worms:
The ups and downs of X-chromosome regulation. Curr. Opin. Genet. Dev. 8: 179-184.

Meller, V.H. and Rattner, B.P. 2002. The roX genes encode redundant male-specific lethal transcripts required for targeting of the MSL complex. EMBO I. 21: 1084-1091.

Oh, H., Bone, J.R., and Kuroda, M.I. 2004. Multiple classes of MSL binding sites target dosage compensation to the X chromosome of Drosophila. Curr. Biol. 14: 481-487.

Ohler, U., Liao, G.C., Niemann, H., and Rubin, G.M. 2002. Computational analysis of core promoters in the Drosophila genome. Genome Biol. 3: RESEARCH0087.

O'Sullivan, J.M., Tan-Wong, S.M., Morillon, A., Lee, B., Coles, J., Mellor, J., and Proudfoot, N.J. 2004. Gene loops juxtapose promoters and terminators in yeast. Nat. Genet. 36: 10141018.

Pokholok, D.K., Harbison, C.T., Levine, S., Cole, M., Hannett, N.M., Lee, T.I., Bell, G.W., Walker, K., Rolfe, P.A., Herbolsheimer, E., et al. 2005. Genome-wide map of nucleosome acetylation and methylation in yeast. Cell 122: 517-527.

Rao, B., Shibata, Y., Strahl, B.D., and Lieb, J.D. 2005. Dimethylation of histone $\mathrm{H} 3$ at lysine 36 demarcates regulatory and nonregulatory chromatin genome-wide. Mol. Cell. Biol. 25: 9447-9459.

Ringrose, L. and Paro, R. 2004. Epigenetic regulation of cellular memory by the polycomb and trithorax group proteins. Annu. Rev. Genet. 38: 413-443.

Ringrose, L., Rehmsmeier, M., Dura, J.M., and Paro, R. 2003. Genome-wide prediction of Polycomb/Trithorax response elements in Drosophila melanogaster. Dev. Cell 5: 759-771.

Rougvie, A.E. and Lis, J.T. 1990. Postinitiation transcriptional control in Drosophila melanogaster. Mol. Cell. Biol. 10: 6041-6045.

Schübeler, D., MacAlpine, D.M., Scalzo, D., Wirbelauer, C., Kooperberg, C., van Leeuwen, F., Gottschling, D.E., O'Neill, L.P., Turner, B.M., Delrow, J., et al. 2004. The histone modification pattern of active genes revealed through genomewide chromatin analysis of a higher eukaryote. Genes \& Dev. 18: 1263-1271.

Sims III, R.J., Belotserkovskaya, R., and Reinberg, D. 2004. Elongation by RNA polymerase II: The short and long of it. Genes \& Dev. 18: 2437-2468.

Smith, E.R., Pannuti, A., Gu, W., Steurnagel, A., Cook, R.G., Allis, C.D., and Lucchesi, J.C. 2000. The Drosophila MSL complex acetylates histone $\mathrm{H} 4$ at lysine 16, a chromatin modification linked to dosage compensation. Mol. Cell. Biol. 20: 312-318.

Smith, E.R., Allis, C.D., and Lucchesi, J.C. 2001. Linking global histone acetylation to the transcription enhancement of Xchromosomal genes in Drosophila males. J. Biol. Chem. 276: 31483-31486.

Straub, T., Gilfillan, G.D., Maier, V.K., and Becker, P.B. 2005. The Drosophila MSL complex activates the transcription of target genes. Genes \& Dev. 19: 2284-2288.

Stuckenholz, C., Kageyama, Y., and Kuroda, M.I. 1999. Guilt by association: Non-coding RNAs, chromosome-specific proteins and dosage compensation in Drosophila. Trends Genet. 15: 454-458.

Turner, B.M., Birley, A.J., and Lavender, J. 1992. Histone H4 isoforms acetylated at specific lysine residues define individual chromosomes and chromatin domains in Drosophila polytene nuclei. Cell 69: 375-384.

Wade, P.A., Pruss, D., and Wolffe, A.P. 1997. Histone acetylation: Chromatin in action. Trends Biochem. Sci. 22: 128132. 


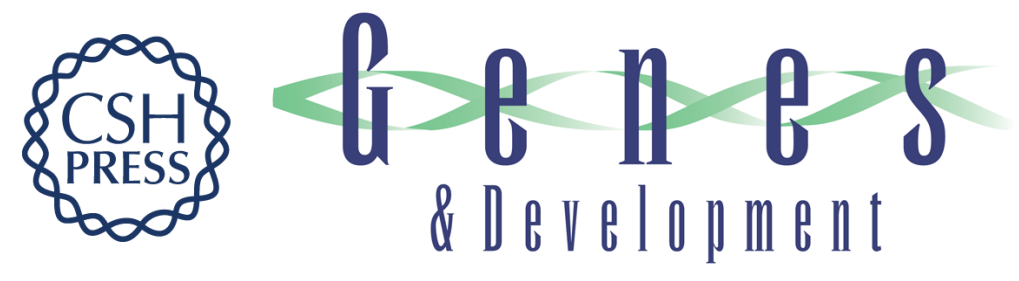

\section{Dosage compensation in high resolution: global up-regulation through local recruitment}

Dirk Schübeler

Genes Dev. 2006, 20:

Access the most recent version at doi:10.1101/gad.1423006

\section{Related Content X-chromosome-wide profiling of MSL-1 distribution and dosage compensation in Drosophila \\ Gaëlle Legube, Shannon K. McWeeney, Martin J. Lercher, et al. Genes Dev. UNKNOWN, 2006 20: 871-883 Chromosome-wide gene-specific targeting of the Drosophila dosage compensation complex \\ Gregor D. Gilfillan, Tobias Straub, Elzo de Wit, et al. \\ Genes Dev. UNKNOWN , 2006 20: 858-870 High-resolution ChIPchip analysis reveals that the Drosophila MSL complex selectively identifies active genes on the male $X$ chromosome \\ Artyom A. Alekseyenko, Erica Larschan, Weil R. Lai, et al. \\ Genes Dev. UNKNOWN , 2006 20: 848-857 \\ References This article cites 36 articles, 13 of which can be accessed free at: http://genesdev.cshlp.org/content/20/7/749.full.html\#ref-list-1 \\ Articles cited in: \\ http://genesdev.cshlp.org/content/20/7/749.full.html\#related-urls \\ License \\ Email Alerting \\ Service \\ Receive free email alerts when new articles cite this article - sign up in the box at the top right corner of the article or click here.}

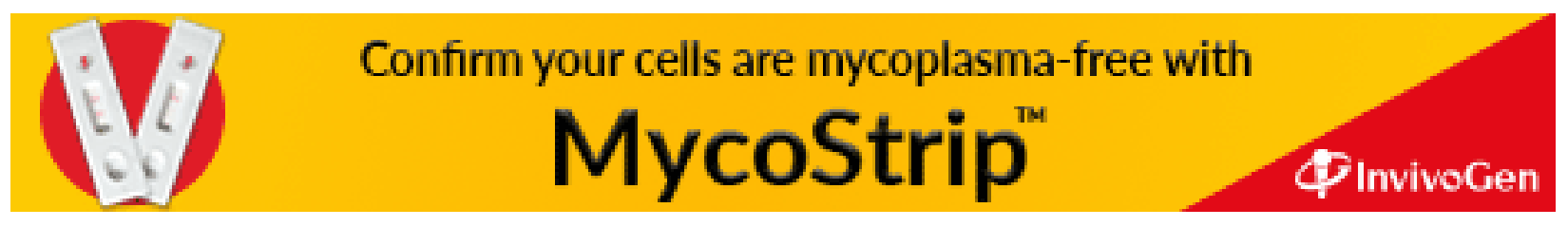

